\title{
At long last: A joint rehabilitation project between Switzerland and Armenia
}

\author{
Twenty years of collaboration between Swiss and Armenian Rehabilitation profes- \\ sionals resulted in two centres in Yerevan. The main focus was education and train- \\ ing of Armenian professionals on site and abroad. Good quality rehabilitation can \\ now be provided but the work is far from done.
}

\author{
Ara S Babloyan ${ }^{a}$, \\ Brigitta B Jann ${ }^{b}$ \\ a Professor of Pediatric Surgery \\ in Yerevan State Medical \\ University, Chairman of \\ Standing Committee on \\ Health Care, Maternity and \\ Childhood in National \\ Assembly of the Republic \\ of Armenia
}

b Retired, former associate professor Emory University SOM, Atlanta, GA, USA, Diplomate of the American Board of PM+R (Physical Medicine and Rehabilitation)

Conflicts of Interest and Source of Funding: This Project was funded by the Swiss foundation «Help for Armenia, Schaffhausen» (HFA) with help from the Swiss office «Direktion für Entwicklung und Zusammenarbeit» (DEZA) and private contributions.

No commercial party having a direct financial interest in the project has or will confer a benefit upon the authors or upon any organisations with which the authors are affiliated.

Correspondance:

Brigitta B Jann, M.D.

Würzenbachstrasse 67

CH-6006 Luzern

brigitta.jann[at]bluewin.ch
Rehabilitation medicine often plays a secondary role in developing countries. In the light of natural disasters, persecution, war and fiscal constraints acute care medicine takes up the available resources and rehabilitation of a people's physically challenged persons cannot be met adequately. Of equal importance are cultural attitudes towards disability. Armenia, a country ravaged by war, genocide and natural disaster, was left in shambles after the collapse of the Soviet Union. Rehabilitation efforts during Soviet time were limited, disabled persons culturally regarded as second class citizens.

WHO in 2006 recognized the need for encouraging the development of rehabilitation services in developing countries and created a medium term plan for the years 2008-20013 [1]. They proposed the following: Raising awareness, building national capacities, supporting national efforts, promoting community-based strategies, production of assistive devices and fostering multisectorial partnerships and networks.

We started pursuing similar paths long before that. Our first efforts go back to 1993.

\section{Current state}

Yerevan, a city of slightly over one million inhabitants, is the capital and largest city of Armenia and one of the world's oldest continuously inhabited cities. An unkind historical past left it struggling to cover its most basic needs and the quality of avail-

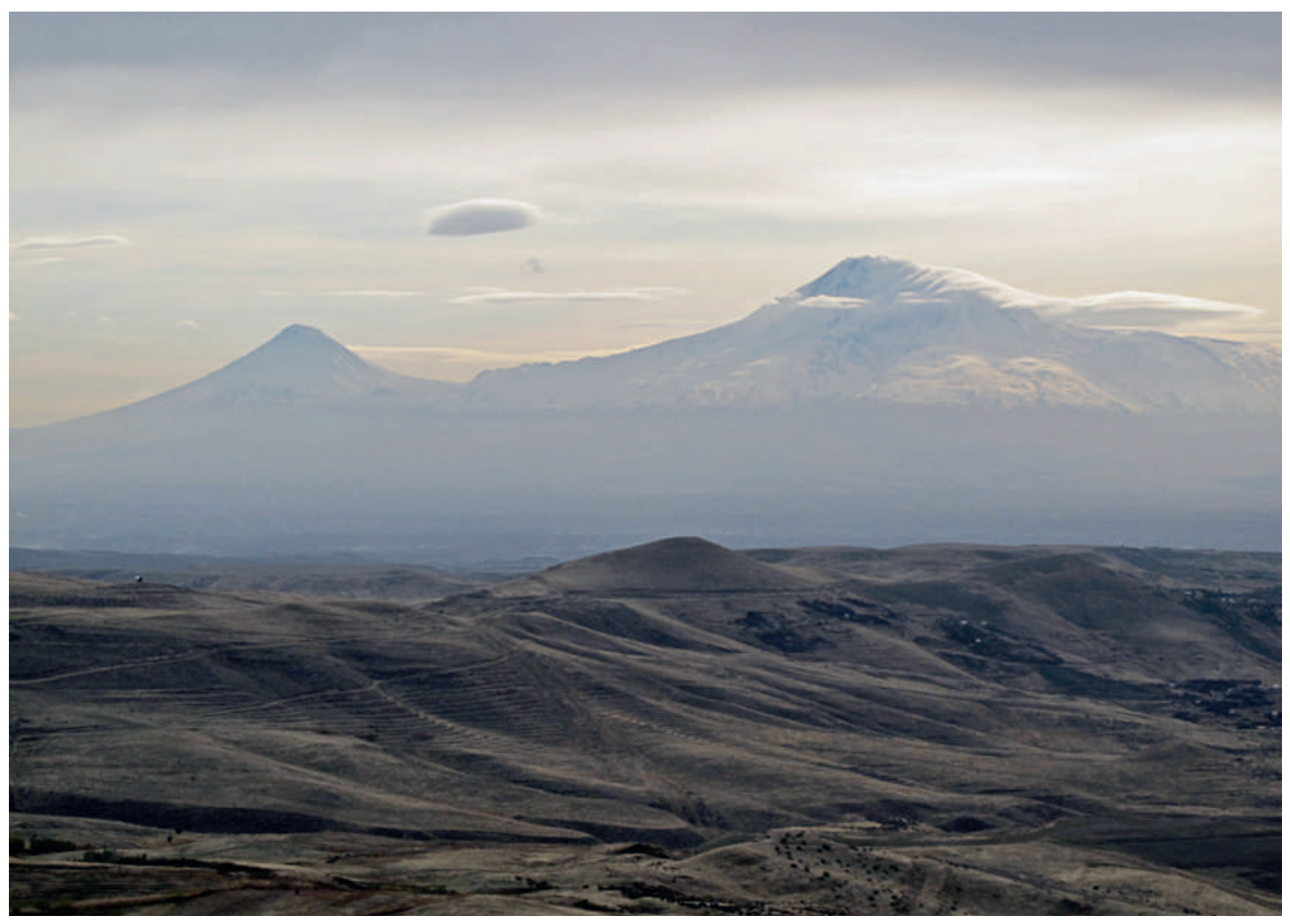

Mount Ararat, a symbol of Armenia and its history, now located in Turkey. (Photography: Meinrad Frei, Schneisingen) 
able medical services, though constantly improving, still requires continuous work and outside help. In the area of rehabilitation services tireless efforts of local and international professionals and outside funding have certainly created centres of good quality treatment.

Republican Children's Rehabilitation Center, (REHAB, a Closed Joint Company, CJC): It is now a regional centre of excellence in Rehabilitation, serving the pediatric and adult population. Originally conceived as a pediatric rehabilitation facility it is now capable to treat individuals of all ages. The diagnoses seen

\section{«Post-Soviet work attitude, low wages and the almost total lack of governmental funding interfered with motivation.»}

range from birth defects, traumatic and nontraumatic brain injuries, neurodegenerative diseases to orthopedic conditions. The centre employs 12 physicians: 6 physiatrists, 2 pediatricians, 1 dentist, 1 gastroenterologist, 1 laboratory physician and 1 electrotherapist. REHAB offers thirty inpatient beds; mother or other family stays with the children. 14 physical therapists (PT), 5 speech and language pathologists (SLP) and 4 psychologists work on site. The physiatrists are by now experienced, well trained, knowledgeable and competent. They also provide consultations in acute care hospitals, most notably Erebuni Hospital, an associated acute care

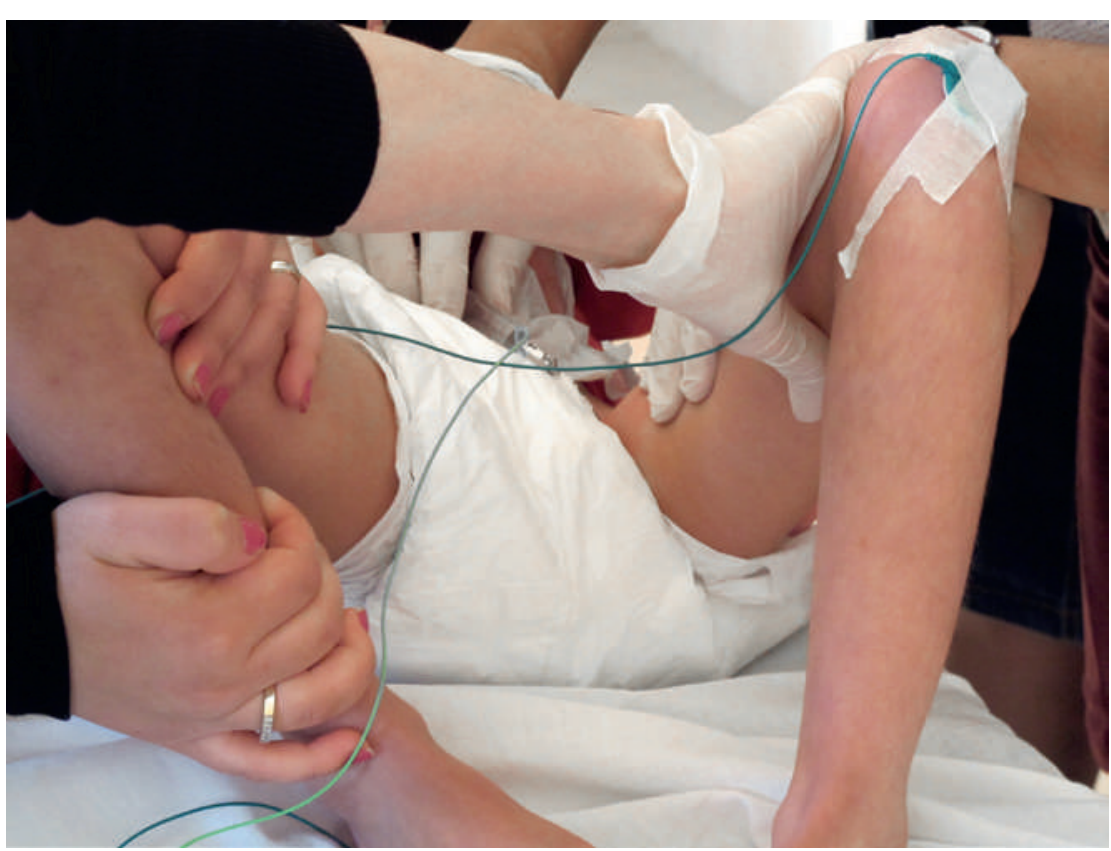

Armenian physicians perform a neurolysis of the N. obturatorius in a child with hip dislocation and severe adductor spasticity. facility across town. The various therapists had ample local and foreign training and most recently the chief physical therapist sustained her certification as an adult Bobath [2] instructor. The centre offers comprehensive in- and outpatient rehabilitation. The expertise of some of the physicians in chemodenervation has made it a centre for spasticity intervention. REHAB has now obtained regional attention and is getting referrals from surrounding countries.

ArBeS (Armenian, Belgian, Swiss) Health care Center (a rehabilitation branch of ARABKIR, United Children's Charity Foundation's Hospital): It is an outpatient facility primarily for pediatric rehabilitation care. Recently it has expanded into adult treatment. The four Physiatrists on the staff have been trained locally and abroad and are competent and knowledgeable. 5 PTs, 3 Occupational Therapists (OT), 5 SLPs, most of them with ample local and foreign training, provide clinical services. In addition, Developmental Medicine is represented by a specialist trained for over a year in the department of Developmental Medicine of the University of Zürich. Uniquely they offer manual therapy services: Two physicians have been trained and certified in Maitland manual therapy [3] and are treating musculoskeletal conditions in children and adults. A first scientific article about their treatment results has recently been published [4]. Two of the physiatrists perform consultations and treatments at Kharbert, a state run orphanage for disabled children in the environment of Yerevan. They are experienced in chemodenervation (see figure). In collaboration with other professionals and institutions they offer comprehensive services to low back pain patients. Training of young rehabilitation physicians is a priority. The therapists too had ample local and foreign training and experience. Of interest is the outreach program that started a few years ago. In cooperation with other agencies, satellite clinics have been established in the country: 7 Child Development and Rehabilitation centres in 6 regions of Armenia. They are run by former resident physicians, providing much needed rehabilitation care to the rural population. For their clinical data collection they have adopted the International Classification of Functioning, Disability and Health (ICF) [5].

Day care (a rehabilitation branch of ARABKIR): A building adjacent to ArBeS houses a day rehabilitation program for autistic children. This is run by a physician with a Master of Philosophy in Special Education (Norway) and 6 SEs have been locally trained by Swiss professionals. The same building also houses an orthotic shop allowing for local manufacturing of assistive devices.

ARABKIR Medical Center - Institute of Child and Adolescent Health: Is the acute care institution associated 
with ArBeS. With financial assistance from DEZA a program has been established and 3 acute care PTs were specifically trained by Swiss PTs. Under the supervision of a physician from ArBeS clinical physical therapy in the acute care is delivered from ICU to discharge.

These centres now have the capability to and actually do train local professionals, offer residency training for physicians, Bobath courses for therapists and nurses. Some of the courses are attended by professionals from neighboring countries.

Professionals from all these centres teach at the local medical university and the institute for physical culture

\section{The beginnings}

The 1988 devastating earthquake brought Armenia suddenly and painfully to the world's attention and help dropped in internationally.

The Swiss Nephrology society responded by sending a specialist in intensive care (Jean-Pierre Bernhardt) and dialysis equipment to the crush victims. He was soon joined by a pediatric nephrologist (Ernst Leumann) from the University of Zürich. The first author, a pediatric surgeon and later minister of health, connected with the two and helped organise the implementation of pediatric Nephrology in Armenia. Thus began a comprehensive, interdisciplinary and successful cooperation between Yerevan and the University Children's Hospital of Zurich that is still ongoing and thriving. It was Leumann's wife Christina, a SLP, who originally spawned the rehabilitation effort and succeeded in bringing in other rehabilitation professionals.

Generally natural disasters leave a myriad of disabilities and need for rehabilitation services behind. The Red Cross (ICRC) built a spinal cord injury centre that developed into a referral facility for the entire region. Unfortunately after cessation of foreign support, the facility had to be closed [6]. Project Health Opportunity for People Everywhere (HOPE, USA) provided training and clinical rehabilitation services with professionals from the US being on site for four years (REHAB). This was a valuable seed for the further development of rehabilitation. However, after cessation of foreign support, this activity decreased as well. As early as 1993, a Swiss PT from the University Children's Hospital in Zürich started treating disabled children and educating professionals in Yerevan. She was soon joined by a SLP and a pediatrician. Verein Armenien Direkt (VAD), a Swiss organisations of mostly volunteers and private sponsors, provided initial funding. Early accommodations and work conditions for these courageous pioneers were rather modest if not uncomfortable. Most of them initially financed their own trips.

\section{Development}

Out of the original efforts grew a multidisciplinary rehabilitation program and in 1998 became an important arm, Pediatric Rehabilitation Neurodevelopmental Training (NDT), of a NGO, the «Foundation Help for Armenia, Schaffhausen» (HFA), who associated with the two centres in Yerevan, REHAB and Arabkir/ArBeS. HFA was very successful in recruiting Swiss professionals from every discipline of pediatric rehabilitation. Several PTs, OTs, SLPs, nurses, SEs, pediatricians, psychiatrists and physiatrists joined the effort and provided repeated on-site clinical services, counseling and training. DEZA cosponsored from the beginning rehabilitation courses and conferences. Due to the efforts of the sponsoring organisations it was soon possible to allow for the conduction of local workshops, repeated visits by Swiss Rehabilitation professionals and sponsorship for the attendance of workshops, courses, practicums of Armenian professionals abroad. Thanks to the generous sponsorship of a pediatrician, one of the originators of infant Bobath therapy [2] (Elsbeth Küng), several Bobath courses were held in Yerevan. We were able to provide much needed equipment and instrumentation to support the clinical ventures. A PT (Erika Hammer) started a Maitland Therapy program [3] and two physicians are now certified in the technique. Cosponsorship of building renovations allowed for an increasingly adequate physical environment. The Swiss professionals of all these subspecialties and representatives from the sponsoring agency met regularly in order to plan priorities at a particular time. The respective Armenian specialists were asked for their input, help and participation in implementing the planned activities. Each subspecialty had an Armenian and Swiss representative jointly responsible for their field. What started initially as a pediatric project was gradually expanded into adult rehabilitation care.

The individual efforts are too many to be listed in detail but they can be divided into the following categories: On-site training, formal courses and clinical services by Swiss professionals, sponsorship of Armenian professional's attendance at courses, international conferences, practicums and internships abroad funding of building renovations, equipment and medication. HFA financed travel expenses, housing and a small daily allowance.

\section{Conclusion}

It would be pretentious to claim that all this evolved totally void of problems and tribulations. The rather strict and rigid Swiss work ethic was difficult to understand and cultural differences proved to be somewhat of a hindrance at times. Post-Soviet work attitude, low wages and the almost total lack of governmental funding interfered with motivation. The awareness of the importance of data collection and documentation needed to be raised. Teaching materials were outdated or absent. Though it took some good twenty years, an atmosphere of mutual toler- 
ance and understanding, the strong will and commitment of our Armenian colleagues and the tireless efforts of the Swiss professionals paid off in the long run and the success of the centres is a definite proof of our collaboration. Good quality rehabilitation can now be provided in Armenia. The local Armenian expertise is sufficient to offer local courses, clinical
Medicine and Rehabilitation $(\mathrm{PM}+\mathrm{R})$ department at the medical university. Associations with outside educational institutions are needed to provide further education and exchange (discussions with a yet not to be named institution are under way). There is ample potential for mutual benefit through exchange of residents and professionals from all disciplines.

\section{«The Armenian professionals now possess marketable skills and are able to generate revenue.»}

education and training. The Armenian professionals now possess marketable skills and are able to generate revenue. The centres enjoy a regional international excellent reputation evidenced by attendance at Armenian educational events and patient referrals from neighboring countries. It proved to be an invaluable experience of friendship and intercultural exchange for the involved Swiss professionals.

\section{Recommendations}

The work is far from done. After discussing future needs with the involved key professionals of both countries we make the following recommendations:

The public image of rehabilitation still needs to improve. Rehabilitation has to become an accepted part of the continuum of care. This requires governmental policies and guidelines and most important governmental financing. Though important, the professionals' increased ability for revenue production is not sufficient for the support of the rehabilitation effort.

Much still is to be done to offer rehabilitation on a community-based level. Social integration, maintenance of affected children in family and school, work integration to name but a few, is all important.

State of the art curricula of education for professionals of all rehabilitation disciplines need to be created, ideally spearheaded by a strong Physical
A local society of $\mathrm{PM}+\mathrm{R}$ incorporating professionals from all rehabilitation disciplines should be formed. Such a society would have the opportunity for local lobbying and international representation and networking.

Acknowledgement: The authors would like to express their appreciation and gratitude to the many members of the teams on both sides, which through their tireless effort and commitment made this possible.

\section{Bibliography}

1 World Health Organisations Disability and Rehabilitation. Global Programming note 2006-2007: Promoting access to healthcare services for persons with disabilities.

2 Bobath K, Bobath B. A treatment of cerebral palsy based on the analysis of the patient's motor behavior. Br J Phys Med. 1952;5:107-17.

3 Maitland GD. Manipulation - Mobilisation. Physiotherapy. 1966;11:382-5.

4 Danielyan M, Jann BB: Maitland manual therapy: a novel approach to treatment of musculoskeletal disorders. Pharma 2013;7:84-7.

5 World Health Organizations Geneva. International Classification of Functioning, Disability and Health. Geneva: Editor WHO; 2011

6 Brunner N. Kinderrehabilitation in Armenien. Schweiz Ärztezeitung. 2007;88:163-5. 